Supporting Information

\title{
Multiplex Biosensing Based on Highly Sensitive Magnetic Nanolabel Quantification: Rapid Detection of Botulinum Neurotoxins A, B, and E
}

Alexey V. Orlov ${ }^{\dagger}$, Sergey L. Znoyko ${ }^{\dagger}$, Vladimir R. Cherkasov ${ }^{\ddagger}$, Maxim P. Nikitin ${ }^{\dagger}$, and Petr I. Nikitin ${ }^{* \uparrow}$

${ }^{\dagger}$ Prokhorov General Physics Institute, Russian Academy of Sciences, 38 Vavilov Street, Moscow, 119991, Russia

${ }^{*}$ Moscow Institute of Physics and Technology, 9 Institutskii per., Dolgoprudny, Moscow Region, 141700, Russia

${ }^{\S}$ National Research Nuclear University MEPhI (Moscow Engineering Physics Institute), 31 Kashirskoe Shosse, Moscow, 115409, Russia

॥ These authors contributed equally to this work
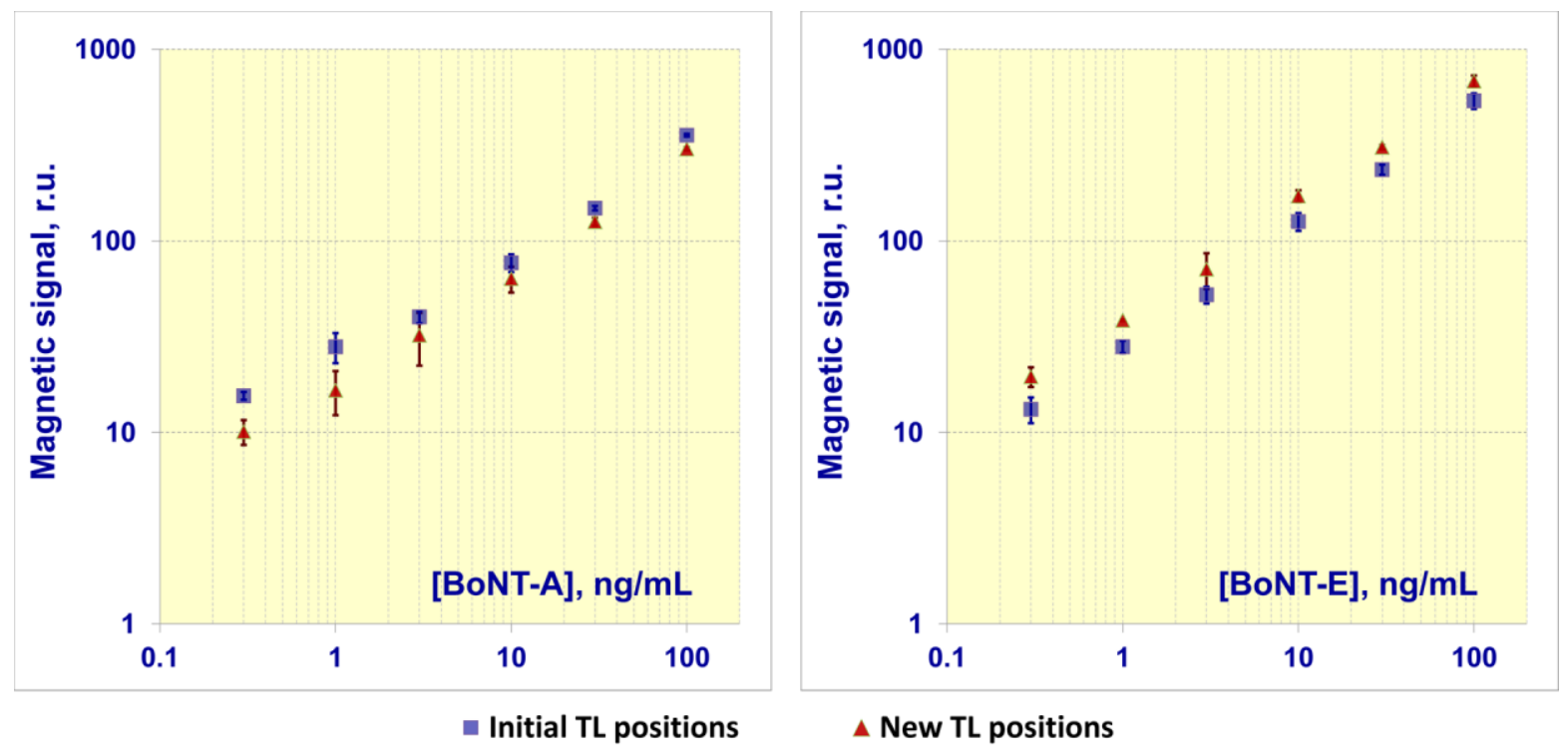

Figure S1. Calibration curves with different TL positions: blue squares - TL at $22 \mathrm{~mm}$; red triangles $\mathrm{TL}$ at $30 \mathrm{~mm}$ for BoNT-A (on the left) and TL at $14 \mathrm{~mm}$ for BoNT-E (on the right)
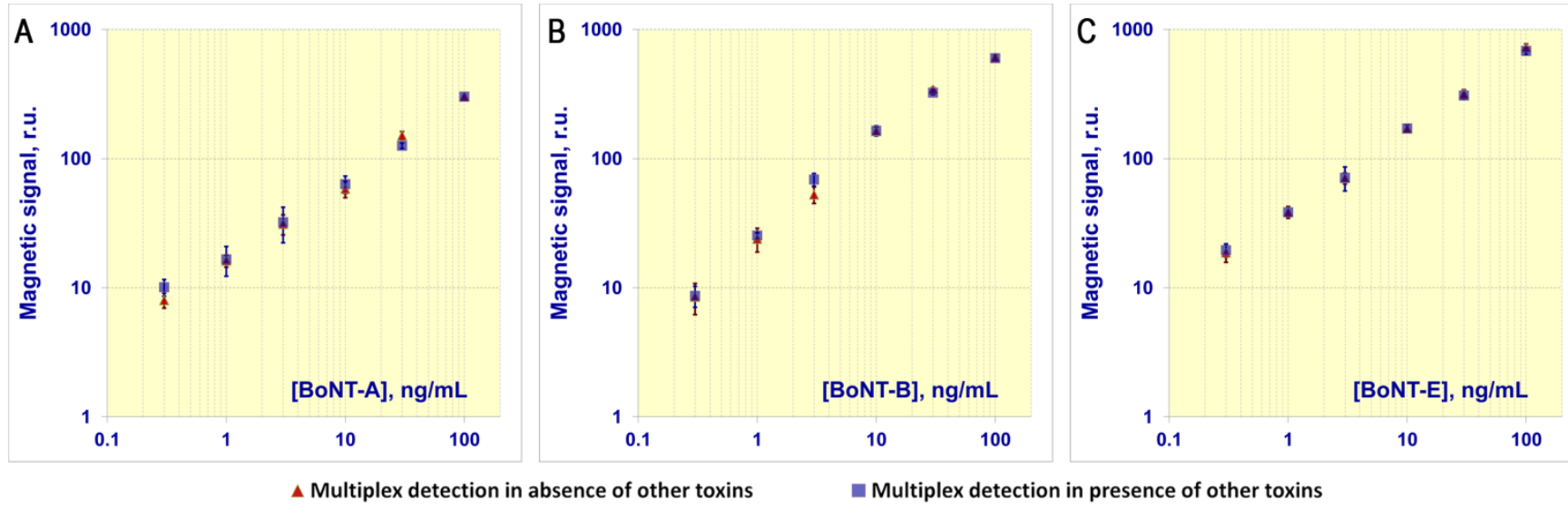

Figure S2. Calibration curves of multiplex detection for every toxin in the presence and absence of other two toxins 